\title{
Single-centre experience and practical considerations of the benefit of a second cochlear implant in bilaterally deaf adults
}

\author{
Dominik Péus ${ }^{1,2}{ }^{(\mathbb{D}} \cdot$ Andreas Pfluger $^{1} \cdot$ Sophia Marie Häussler ${ }^{1} \cdot$ Steffen Knopke $^{1} \cdot$ Manuel Christoph Ketterer $^{1}(\mathbb{D}$. \\ Agnieszka J. Szczepek ${ }^{1} \cdot$ Stefan Gräbel $^{1} \cdot$ Heidi Olze ${ }^{1}$ (I)
}

Received: 31 March 2020 / Accepted: 20 August 2020 / Published online: 5 September 2020

(c) The Author(s) 2020

\begin{abstract}
Purpose Bilateral cochlear implant (CI) implantation is increasingly used in the auditory rehabilitation of bilaterally deafened adults. However, after successful unilateral implantation, objective patient counselling is essential.

Methods We investigated the extra benefit of a second CI in adults in terms of health-related quality of life, tinnitus, stress, anxiety, depression, quality of hearing, and speech recognition. Hearing ability was assessed by using the Freiburg monosyllable speech discrimination test (FB MS) and the Oldenburg sentence test with azimuth variations. In a prospective patient cohort, we administered validated questionnaires before a CI, after a first CI and after a second CI implantation.

Results The study included 29 patients, made up of nine women and 20 men. The median time between the first and the second implantation was 23 months. The mean total NCIQ score and TQ before a CI improved significantly after both implantations. Stress, anxiety, and depression were stable over time and were not significantly affected by CI implantations. Speech recognition with noise significantly improved after the first and again after the second CI. Correlation analysis showed a strong connection between auditory performance and HRQoL.

Conclusion We demonstrated that a unilateral CI benefitted many fields and that the second sequential CI leads again to additional improvement. Bilateral CI implantation should, therefore, be the standard form of auditory rehabilitation in deafened adults.
\end{abstract}

Keywords Cochlear implant · CI · Bilaterally deafened · Hearing loss · Quality of life · Speech recognition

\section{Introduction}

The number of bilaterally deafened adults is increasing, mainly due to a constantly ageing society. A bilateral CI is a widely accepted medical practice in infants and children $\mathrm{h}$ [1]. However, in post-lingual bilaterally deafened adults, there is ongoing discussion regarding outcomes as well as medical and surgical safety concerns [2]. It is known that CIs allow people with hearing disorders to regain auditory perception and an acoustic understanding of speech. Among the positive consequences of a bilateral CI versus a unilateral

Heidi Olze

heidi.olze@charite.de

1 Department of Otorhinolaryngology, Campus Virchow Klinikum, Charité-Universitätsmedizin Berlin, Augustenburger Platz 1, 13353 Berlin, Germany

2 Department of Otorhinolaryngology, Kantonsspital Baselland, Liestal, Switzerland
CI are improved sound localisation, enhanced speech perception, and a reduced risk of being "off-air" [3-5]. The theoretically potential disadvantages include bilateral vestibular alterations, surgical complications, and cost [6-8]. The debate about cost-effectiveness is particularly controversial [9-11]. Up to this point, most research investigating bilateral CI supply has focused on auditory improvement, including sound localisation and enhanced speech perception, but little is known about the subjectively experienced changes created by a CI, and very few studies have analysed the health-specific quality of life changes due to a second CI $[12,13]$.

The list of indications for cochlear implantation has expanded in the last decade. As a result, increased numbers of adult post-lingually deafened patients are scheduled for a second CI. To measure the impact of CIs on patients' lives, our study group uses a test battery, which in addition to the auditory performance, assesses the subjective perception of stress factors, patients' resources, ways of coping with 
stress, and the degree of anxiety and depressive symptoms [14]. In the past, the benefit of the second CI was investigated in a retrospective manner, which had some limitations, because the patients had to reply to the questionnaires retrospectively, and the period between implantation and questionnaire replies was quite heterogeneous. In the meantime, the test battery was refined and is routinely used to assess the patients with asymmetric hearing loss, after a positive impact of the first CI. The present study was designed as a prospective cohort study and aimed to investigate the impact of a second CI on the auditory performance, perceived stress, patients' resources, coping, and the degree of anxiety and depressive symptoms of implanted subjects.

\section{Methods}

\section{Patients}

Between July 2010 and May 2016, 29 bilaterally deaf adult patients who were scheduled for a bilateral CI implantation were included in this study. The implantations were performed sequentially, and the median time between the implantations was 23 months. Patients were interviewed before the first implantation and six months after the first and the second implantation.

The cohort consisted of nine women and 20 men, averaging 57.63 years of age. The period of deafness was determined based on the reported time when a patient felt that wearing a hearing aid had stopped being of significant benefit, and averaged 18.92 years. The mean value Freiburg monosyllable speech discrimination test (FB MS) for implanted ears was 6.05 ( $\mathrm{SD}=11.85)$, while on contralateral ears, it was $10.87(\mathrm{SD}=16.42)$.

\section{Variables and goals}

For each patient, we collected data at three time points: prior to the first CI, six months after the first implantation, and again six months after second implantation. Patients filled paper questionnaires, as described by Bruggemann et al. (2017), including the Nijmegen Cochlear Implantation Questionnaire (NCIQ), the Tinnitus Questionnaire (TQ), the Perceived Stress Questionnaire (PSQ), the brief German COPE (COPE), the General Depression Scale (ADS), and the Generalised Anxiety Disorder (GAD) [15-22]. Subjective hearing ability was assessed using the Oldenburg Inventory, and audiometric measurements were performed in the audiometry unit of the otolaryngology department.

Speech perception was measured in all patients before the $\mathrm{CI}$, using the FB MS. This involved presenting patients with $2 \times 20$ monosyllabic words at a volume of $65 \mathrm{~dB}$ HL in quiet conditions. After the first and second CIs, the FB MS and
Oldenburger Satz test (OLSA) were used [21]. The OLSA consists of a five-word sentence used to assess speech perception in noisy conditions and is defined as a signal-to-noise ratio in dB SPL (SNR). The OLSA was used in the following five test conditions: after the first CI, (1) speech and noise were presented from the front, (2) noise was directed at the implanted ear and speech was directed at the deaf ear and (3) vice versa. After a second CI, (4) speech and noise were presented from the front and (5) speech was directed at the first implanted ear, while noise was directed at the second implanted ear. Azimuth variation for speech and noise presentation simulates an everyday hearing situation.

The primary objective of this prospective analysis was to identify predictive values for a successful second CI, defined by an increase in health-related quality of life and better performance in the monosyllable test and the more difficult speech perception tests.

\section{Statistical procedure}

Twenty-nine patients were included in this study. Statistical evaluation was carried out using the software program SPSS (IBM Corp. Released 2017. IBM SPSS Statistics for Windows, Version 25, Armonk, NY: IBM Corp.). Frequency tables, mean values, and standard deviations were calculated descriptively. The Kolmogorov-Smirnov test was used to test for distribution normality, and the Wilcoxon test was used to test the significance of differences before and following implantation. Although some quantitative data were available, there were insufficient data for regression analysis, and so we report effect sizes herein, using Pearson's correlation test. Significance was set at $p<0.05$.

\section{Results}

\section{Additional health-related quality of life}

The disease-specific quality of life measured by the NCIQ improved significantly after the first $\mathrm{CI}$ and again after the second CI. The mean total NCIQ score before any CI was $42.80 \pm 17.84$. After a first CI, it improved to $55.66 \pm 16.06$ $(p<0.000)$ and then improved further to $63.28 \pm 18.53$ ( $p=0.012$ ) following the second CI (see Fig. 1a). NCIQ subdomains, including basic sound perception, advanced sound perception, speech production, self-esteem, and social interactions, significantly improved after both CIs, except for subdomain NCIQ 3, i.e. speech production (see Table 1).

\section{Audiological improvement-unilateral versus bilateral $\mathrm{CI}$}

The mean value FB MS for the first implanted ears was $6.05 \pm 11.85$; on contralateral ears, it was $10.87 \pm 16.42$. 
Fig. 1 Changes in mean total scores for NCIQ, TQ, OI, PSQ, GAD-7, and ADS prior to a CI, after a first $\mathrm{CI}$ and after a second CI. Changes to the NCIQ, TQ, OI, and PSQ subscales behaved similarly to the mean total scores and were therefore not plotted. Error bars: 95\% confidence interval. ${ }^{*} p<0.05$; $* * p<0.01 ; * * * p<0.001$
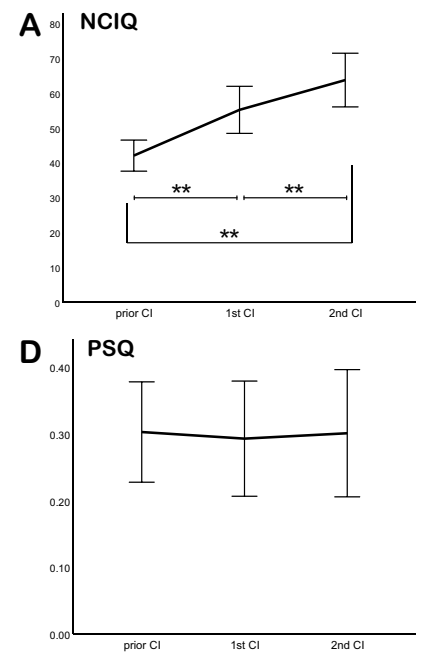
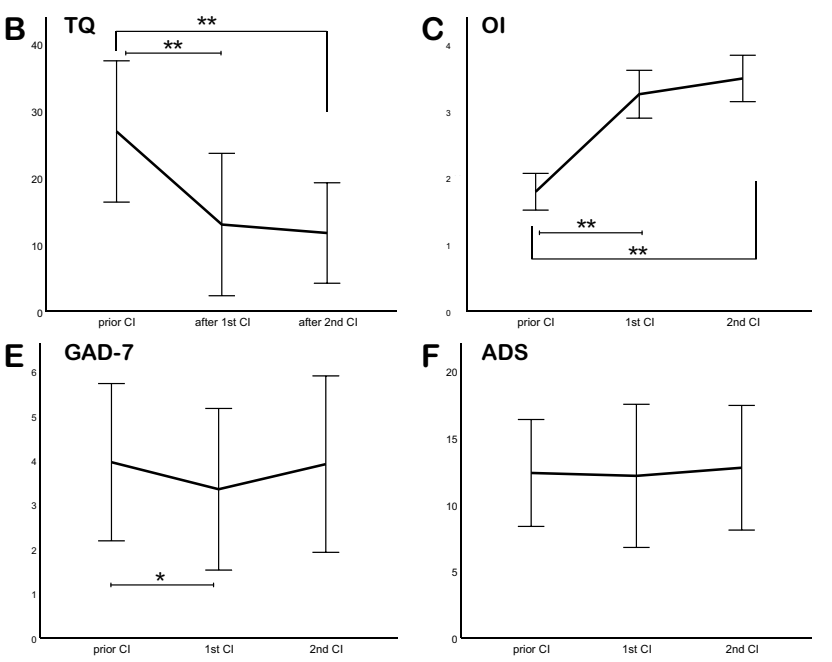

After first CI, FB MS increased to $48.69 \pm 28.94(p<0.001)$. After the second CI, the FB MS improved further to $66.43 \pm 19.57(p=0.002)$ with bilaterally active CI. After the second $\mathrm{CI}$ operation, both ears were also investigated with only one active CI. The second operated ear performed slightly better $56.59 \pm 19.24$ versus the first operated ear $50.68 \pm 22.16$. However, the difference was not statistically significant.

When speech and noise were presented from the front, the mean OLSA SNR was $0.368 \pm 3.109 \mathrm{~dB}$ SPL with a bilateral CI, while the mean OLSA SNR was $1.881 \pm 4.459 \mathrm{~dB}$ SPL with a unilateral CI. The $1.513 \mathrm{~dB}$ SPL difference was significant $(p=0.002)$. If the speech was presented to the first implanted ear, and noise to the deaf ear, the mean OLSA SNR was $-4.241 \pm 5.338 \mathrm{~dB}$ respective to the contralateral CI $-3.518 \pm 5.00 \mathrm{~dB}$ SPL. The difference, in this case, was not significant $(p=0.115)$. Speech understanding measures were justifiably the lowest observed when noise was presented to the $\mathrm{CI}$ ear and speech to the deaf ear at $7.231 \pm 4.65 \mathrm{~dB}$ SPL in unilaterally implanted patients.

\section{Subjective hearing ability}

The first CI resulted in a significant improvement in the mean total OI score. Before a first CI, the mean total OI score was $1.81 \pm 0.55$, but this improved to $3.22 \pm 0.55$ after the first CI. All three subdomains, "OI quiet", "OI noise interference", and "OI directional listening", significantly improved, and further progress after a second CI was observed in all OI subdomains.

\section{Tinnitus annoyance}

In the study cohort, tinnitus annoyance was reduced by the first CI to a low level and declined even further after secondary surgery. Twenty-one patients (72\%) reported tinnitus of

some kind before CI implantation, with a mean total TQ score of $27.52 \pm 19.67$. TQ decreased after the first CI to $15.68 \pm 20.37(p=0.004)$ and decreased further after the second to $11.00 \pm 13.44(p=0.041)$. Before any CI, five patients (23.8\%) reported decompensated tinnitus (total TQ score $>46$ ). After the first CI, two patients in the decompensated tinnitus subgroup achieved intermediate levels (total TQ score 31-46). One patient reported a complete absence of tinnitus, and two reported continuous high tinnitus annoyance. These two patients profited from the second CI, with one achieving low-level and the other intermediate-level tinnitus. Each of the three patients (14\%) with intermediate tinnitus annoyance before the first $\mathrm{CI}$ reported a decrease to low tinnitus levels (total TQ score 0-30) after the procedure, and this reduction persisted after the second CI. All subscales scores, including emotional and cognitive distress, intrusiveness, auditory perceptual difficulties, sleeping disturbances, but somatic complaints, declined significantly after the first $\mathrm{CI}$ and again after the second CI (see Table 1).

\section{Psychosocial burden}

To measure the psychosocial burden of CI patients, PSQ, COPE, GAD 7, and ADS questionnaires were filled by the patients and analysed by qualified staff. The perceived stress questionnaire showed relatively low levels of preoperative stress, with a mean total PSQ of $0.31 \pm 0.17$. PSQ total scores remained stable over time. Only the PSQ subscale demands demonstrated a significant reduction after the first CI $(p=0.037)$, and this value remained stable after the second procedure (see Fig. 1d), thereby implying that neither surgery increased patients' stress.

The COPE questionnaire has four subdomains, namely avoidance, seeking support, positive thinking, and active problem-solving. COPE scores have not changed significantly after the first CI. However, significant reductions 


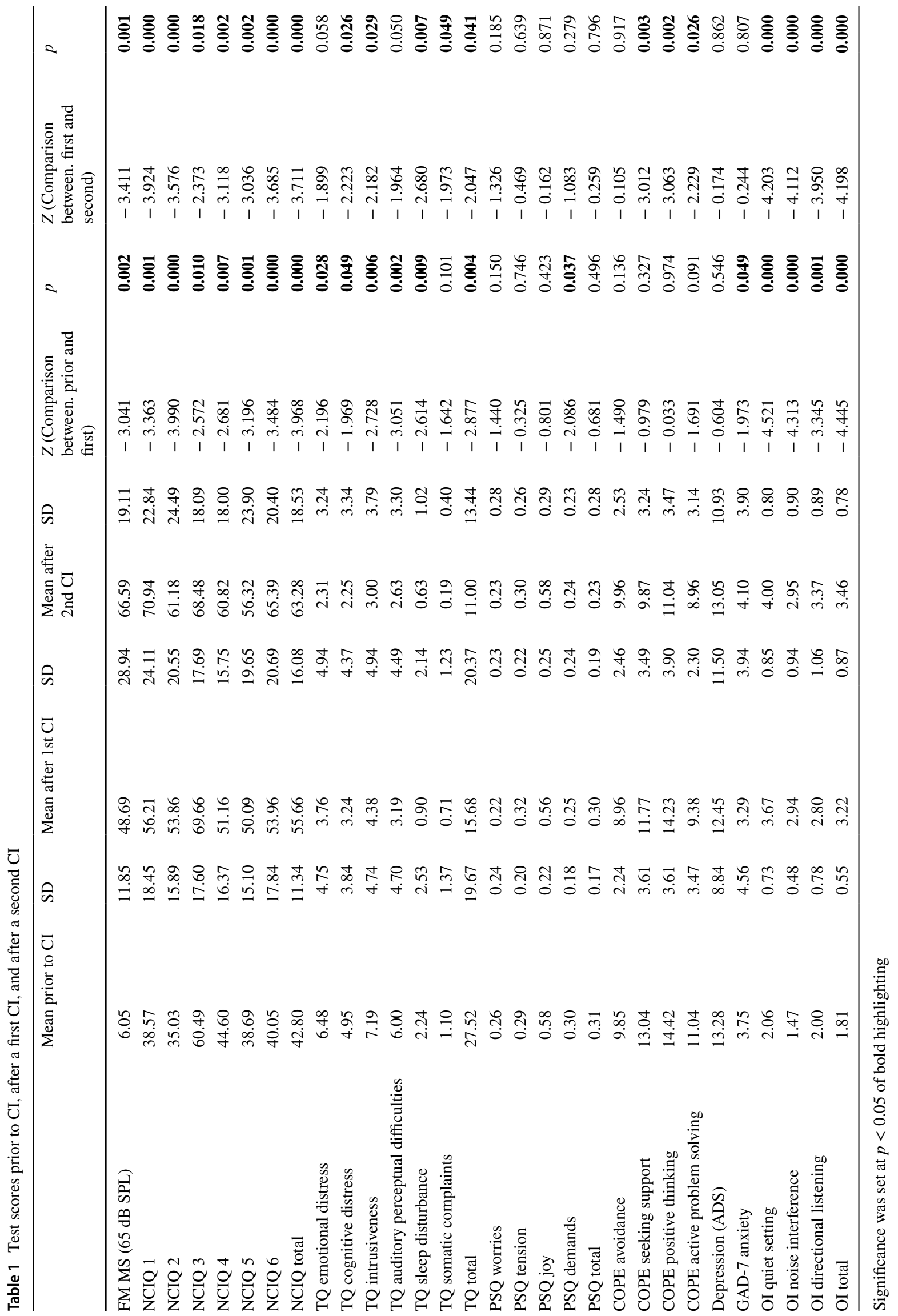


in three of the four subdomains (avoidance, seeking support, and positive thinking) were observed after the second implantation (see Table 1). That may be because coping and problem-solving strategies are less essential after a second CI, thus removing some of the limitations of monaural hearing.

The mean score of the ADS depression questionnaire was $13.28 \pm 8.84$ before any CI, and 10 of the 29 (34.4\%) patients scored 16 or more, which is a degree of depression that is understood to be deserving clinical treatment. There was a slight reduction after the first CI, and a very slight increase after second CI. However, these changes were not significant ( $p=0.546$ and $p=0.862)$.

The mean of the GAD-7 questionnaire, which assesses fear, was $3.75 \pm 4.56$ pre-CI, which indicates minimal to mild anxiety. After the first CI, the mean GAD-7 declined to $3.29 \pm 3.94(p=0.049)$. After the second $\mathrm{CI}$, it was $4.10 \pm 3.90(p=0.807)$. Before CI, nine of the $29(31 \%)$ patients reported at least mild anxiety (4-9 pt.) [20], and this has not changed after either CI.

\section{Correlation of health-related quality of life, psychosocial burden, and hearing}

Pearson correlations were performed in order to gain a better understanding of the relations between HRQoL (NCIQ), tinnitus distress (TQ), stress (PSQ), generalised anxiety disorder (GAD-7), depression (ADS), coping (COPE), subjective quality of hearing (OI), and hearing performance with background noise. The heat maps summarise the correlations after the first and after the second CI (see Fig. 2). For the sake of clarity, we used two mean total scores for NCIQ, TQ, PSQ, GAD-7, and ADS, namely the COPE subscale "seeking support" and the signal-to-noise ratio presented from the front. The first main finding is that after the first $\mathrm{CI}$, tinnitus distress correlated significantly with subjective hearing ability and speech recognition, and after the second $\mathrm{CI}$, tinnitus distress significantly decreased and the previous correlation disappeared. The second main finding is that the correlation between HRQoL and subjective hearing ability is quite strong (Pearson correlation coefficient $=0.543$ ) and becomes even more significant after the second CI (Pearson correlation coefficient $=0.917$ ), highlighting the importance of sufficient hearing capacity for a good quality of life.

\begin{tabular}{|c|c|c|c|c|c|c|c|c|}
\hline \multicolumn{9}{|c|}{ correlations after first $\mathrm{Cl}$} \\
\hline & $\mathrm{NCIQ}$ total & TQ total & Ol total & PSQ total & ADS & GAD-7 & COPE & OLSA \\
\hline health-related quality of life & 1 & -0.362 & 0.543 & -0.360 & -0.444 & -0.288 & 0.028 & -0.254 \\
\hline tinnitus distress & TQ total & 1 & -0.836 & -0.048 & 0.138 & -0.225 & 0.102 & 0.716 \\
\hline subjective hearing ability & & Ol total & 1 & -0.105 & -0.263 & 0.074 & -0.203 & -0.687 \\
\hline stress & & & PSQ total & 1 & 0.817 & 0.836 & 0.482 & -0.171 \\
\hline depression & & & & ADS & 1 & 0.84 & 0.306 & -0.003 \\
\hline generalized anxiety disorder & & & & & GAD-7 & 1 & 0.249 & -0.284 \\
\hline coping seeking support & & & & & & COPE & 1 & -0.116 \\
\hline speech recognition in noise & & & & & & & OLSA & 1 \\
\hline
\end{tabular}

\begin{tabular}{|c|c|c|c|c|c|c|c|c|}
\hline \multicolumn{9}{|c|}{ correlations after second $\mathrm{Cl}$} \\
\hline & NCIQ total & TQ total & Ol total & PSQ total & ADS & GAD-7 & COPE & OLSA \\
\hline health-related quality of life & 1 & 0.011 & 0.917 & -0.483 & -0.449 & -0.397 & $\begin{array}{ll}-0.086 \\
\end{array}$ & -0.426 \\
\hline tinnitus distress & $\mathrm{TQ}$ total & 1 & -0.030 & -0.273 & -0.229 & -0.378 & 0.120 & 0.331 \\
\hline subjective hearing ability & & Ol total & 1 & -0.278 & -0.216 & -0.182 & -0.026 & -0.459 \\
\hline stress & & & PSQ total & 1 & 0.846 & 0.865 & 0.141 & -0.173 \\
\hline depression & & & & ADS & 1 & 0.885 & 0.234 & -0.090 \\
\hline generalized anxiety disorder & & & & & GAD-7 & 1 & 0.222 & -0.293 \\
\hline coping seeking support & & & & & & COPE & 1 & 0.058 \\
\hline speech recognition in noise & & & & & & & OLSA & 1 \\
\hline
\end{tabular}

Fig. 2 Correlation heat map between health-related quality of life, psychosocial burden, subjective quality of hearing, and speech recognition. Pearson correlation coefficients after the first and second
CIs. Significant correlations are highlighted in bold $(p<0.05)$. Green boxes refer to positive correlations. Red boxes refer to negative correlations 


\section{Discussion}

In the present work, we used a prospective study to follow 29 post-lingually bilaterally deaf patients from the moment when they were scheduled for the first cochlear implantation, up to six months after the second cochlear implantation. At present, there is an ongoing global discussion about bilateral cochlear implantation as a standard procedure for bilaterally deafened people. Apart from medical and audiological issues, this discussion involves health politics. Here, we provide evidence about the medical, psychological, and social benefits in patients who were bilaterally, subsequently implanted with CI.

One of our main findings was that HRQoL significantly improved, not only after the first CI implantation but also after the second CI. This remarkable additional improvement was seen in all subdomains of the NCIQ: primary sound perception, advanced sound perception, speech production, self-esteem, activity, and social interactions. Our results corroborate these of others $[10,12,23]$ and fully justify the medical effort.

In addition to the quality of life, speech comprehension, and directional hearing improved after first and after second CI implantation, in agreement with already published data [3]. Also, the quality of life and subjective hearing ability strongly correlated. All three subscales and the mean total score for subjective hearing ability OI (quiet setting, noise interference, and directional listening) improved mainly after the first and to a lesser extent after the second CI (Fig. 1c) - in contrast to NCIQ (Fig. 1a), where a steeper graphical incline was observed. HRQoL strongly correlated with subjective hearing ability, whereas it did not correlate with SNR performance after the first CI, but it did so after the second CI. Therefore, to assess the psychosocial changes caused by a second CI, questionnaires are a useful augmentation to standard speech perception tests and subjective hearing assessment.

Many studies report improved speech perception-and notably, directional listening-after a second CI [12, 24]. The OI subdomain of directional listening improved relative to the other two subdomains, most of all following the second CI in our study, which seems logical, because two functional hearing organs are required for the downstream central auditory pathways to achieve the spatial localisation of sound sources [25]. Interestingly, average head shadow effect in the monaural condition was similar to a bilaterally active CI, which might be explained by temporal and spectral differences of spatially different speech and noise sources (the squelch effect), but electronic noise filters and different CI cues may also contribute in this regard [26]. In the unilateral CI situation, with noise presented to the CI ear and speech to the deaf ear, the mean
SNR was $7.231 \pm 4.65 \mathrm{~dB}$ SPL. An SNR value of $7.231 \mathrm{~dB}$ SPL (different to a bilateral CI by $10.749 \mathrm{~dB}$ SPL) indicates inferior speech recognition. However, the quantitative audiometric measurements correlated only moderately with statistical significance in the binaural situation but correlated better with subjective hearing ability.

Evidence accumulates about the reduction in tinnitus annoyance being closely related to the improvement in HRQoL [27-29]. In the present study, although the patients with decompensated tinnitus benefitted in particular from a second CI, there was no statistically significant correlation between NCIQ and TQ. In the previous data published by Knopke et al. (2017), tinnitus was an inclusion criterion, and there was a higher proportion of patients with decompensated tinnitus. In the present study, the study sample was relatively small, and the patients had relatively low tinnitus annoyance, which might explain our results. However, there was a strong negative correlation between tinnitus distress and subjective hearing ability. In summary, tinnitus reduction after second cochlear implantation does not account for the improvement in the quality of life.

We also demonstrated that the subjective ability of hearing correlates positively with the HRQoL and negatively with elevated scores in the depression assessment (see also Fig. 2). This supports the importance of hearing ability in patients' subjectively experienced HRQoL. Although the information about a type of specific treatment for depression was not assessed, cochlear implantation likely enabled the patients to attend conversational psychotherapeutic therapy.

Before CI, nine of 29 (31\%) patients reported at least mildly severe anxiety [20], which negatively correlated with the quality of hearing and support-seeking. As the hearing performance improved, this correlation became insignificant.

A great deal of effort has been spent on identifying the predictive values for the outcome of a second CI. In summary, no feature of an individual can predict the outcome-even advanced age, a long duration of deafness, or a long interval between implantations are not significant negative predictors [30]. However, all patients prefer to implant the poorer ear first (in many cases, the ear that was suppressed over many years), and they wait until the second ear worsens. This fact produces an analytical heterogeneity. Additionally, the current study could not find predictive values by using regression analysis. The improvement in the HRQoL after the second CI might also be explained as a result of the better ear and upstream connections being suppressed over a shorter period, and therefore hearing performance and experience after the second $\mathrm{CI}$ being judged by $\mathrm{CI}$ recipients as very satisfactory. 


\section{Conclusion}

The present study demonstrates that in bilaterally deafened adult patients, a second CI implantation improves HRQoL, tinnitus annoyance, speech comprehension under noisy conditions, and subjective quality of hearing compared to a one-sided CI condition. The extent of improvement in auditory performance was positively correlated with the improved health-related quality of life. No effect of implantation on depression, stress, and anxiety disorders was observed.

There are no measures currently available after fitting the first CI that help predict whether a patient is a suitable candidate for a second $\mathrm{CI}$; however, nearly all patients profit from a second CI in several respects. Specialists should thus actively talk with patients about a second CI in unilateral CI recipients to inform them objectively about the risks and possible benefits of the second implantation. A bilateral CI implantation should be encouraged in most cases as a standard form of auditory rehabilitation in deafened children and adults-with very few exceptions.

Funding Open Access funding provided by Projekt DEAL.

\section{Compliance with ethical standards}

Conflict of interest None of the authors have a personal conflict of interest to declare.

Ethical approval The Charité ethics committee (Reg.nr. EA2/030/13) approved the study, and all investigations were conducted according to the principles expressed in the Declaration of Helsinki.

Informed consent All patients gave their informed written consent.

Open Access This article is licensed under a Creative Commons Attribution 4.0 International License, which permits use, sharing, adaptation, distribution and reproduction in any medium or format, as long as you give appropriate credit to the original author(s) and the source, provide a link to the Creative Commons licence, and indicate if changes were made. The images or other third party material in this article are included in the article's Creative Commons licence, unless indicated otherwise in a credit line to the material. If material is not included in the article's Creative Commons licence and your intended use is not permitted by statutory regulation or exceeds the permitted use, you will need to obtain permission directly from the copyright holder. To view a copy of this licence, visit http://creativecommons.org/licenses/by/4.0/.

\section{References}

1. Balkany T, Hodges A, Telischi F et al (2008) William house cochlear implant study group: position statement on bilateral cochlear implantation. Otol Neurotol 29(2):107-108. https://doi. org/10.1097/mao.0b013e318163d2ea
2. Basura GJ, Eapen R, Buchman CA (2009) Bilateral cochlear implantation: current concepts, indications, and results. Laryngoscope 119(12):2395-2401. https://doi.org/10.1002/lary.20751

3. Laske RD, Veraguth D, Dillier N et al (2009) Subjective and objective results after bilateral cochlear implantation in adults. Otol Neurotol 30(3):313-318. https://doi.org/10.1097/ MAO.0b013e31819bd7e6

4. Dunn CC, Noble W, Tyler RS et al (2010) Bilateral and unilateral cochlear implant users compared on speech perception in noise. Ear Hear 31(2):296-298. https://doi.org/10.1097/AUD.0b013 e3181c12383

5. Avan P, Giraudet F, Büki B (2015) Importance of binaural hearing. Audiol Neurootol 20(Suppl 1):3-6. https://doi.org/10.1159/00038 0741

6. Wagner JH, Basta D, Wagner F et al (2010) Vestibular and taste disorders after bilateral cochlear implantation. Eur Arch Otorhinolaryngol 267(12):1849-1854. https://doi.org/10.1007/s0040 5-010-1320-1

7. Brown KD, Balkany TJ (2007) Benefits of bilateral cochlear implantation: a review. Curr Opin Otolaryngol Head Neck Surg 15(5):315-318. https://doi.org/10.1097/MOO.0b013e3282ef3d3 $\mathrm{e}$

8. Hansel T, Gauger U, Bernhard N et al (2018) Meta-analysis of subjective complaints of vertigo and vestibular tests after cochlear implantation. Laryngoscope. https://doi.org/10.1002/lary.27071

9. Bichey BG, Miyamoto RT (2008) Outcomes in bilateral cochlear implantation. Otolaryngol Head Neck Surg 138(5):655-661. https ://doi.org/10.1016/j.otohns.2007.12.020

10. Theriou C, Fielden CA, Kitterick PT (2019) The cost-effectiveness of bimodal stimulation compared to unilateral and bilateral cochlear implant use in adults with bilateral severe to profound deafness. Ear Hear. https://doi.org/10.1097/AUD.0000000000000727

11. Foteff C, Kennedy S, Milton AH et al (2016) Cost-utility analysis of cochlear implantation in australian adults. Otol Neurotol 37(5):454-461. https://doi.org/10.1097/MAO.0000000000000999

12. McRackan TR, Bauschard M, Hatch JL et al (2018) Meta-analysis of quality-of-life improvement after cochlear implantation and associations with speech recognition abilities. Laryngoscope 128(4):982-990. https://doi.org/10.1002/lary.26738

13. Olze H, Grabel S, Haupt $\mathrm{H}$ et al (2012) Extra benefit of a second cochlear implant with respect to health-related quality of life and tinnitus. Otol Neurotol 33(7):1169-1175. https://doi.org/10.1097/ MAO.0b013e31825e799f

14. Bruggemann P, Szczepek AJ, Klee K et al (2017) In patients undergoing cochlear implantation, psychological burden affects tinnitus and the overall outcome of auditory rehabilitation. Front Hum Neurosci 11:226. https://doi.org/10.3389/fnhum.2017.00226

15. Fliege H, Rose M, Arck P et al (2005) The perceived stress questionnaire (PSQ) reconsidered: validation and reference values from different clinical and healthy adult samples. Psychosom Med 67(1):78-88. https://doi.org/10.1097/01.psy.0000151491.80178 .78

16. Hinderink JB, Krabbe PF, van den Broek P (2000) Development and application of a health-related quality-of-life instrument for adults with cochlear implants: the Nijmegen cochlear implant questionnaire. Otolaryngol Head Neck Surg 123(6):756-765. https://doi.org/10.1067/mhn.2000.108203

17. Goebel G, Hiller W (1994) Tinnitus-Fragebogen (TF). Standardinstrument zur Graduierung des Tinnitusschweregrades. Ergebnisse einer Multicenterstudie mit dem Tinnitus-Fragebogen (TF) (The tinnitus questionnaire. A standard instrument for grading the degree of tinnitus. Results of a multicenter study with the tinnitus questionnaire). HNO 42(3):166-172

18. Mohiyeddini C, Hautzinger M, Bauer S (2002) Eine LatentState-Trait-Analyse zur Bestimmung der dispositionellen und zustandsbedingten Anteile dreier Instrumente zur Erfassung von 
Depressionen: ADS. BDI und SDS Diagnostica 48(1):12-18. https://doi.org/10.1026//0012-1924.48.1.12

19. Knoll N, Rieckmann N, Schwarzer R (2005) Coping as a mediator between personality and stress outcomes: a longitudinal study with cataract surgery patients. Eur J Pers 19(3):229-247. https:// doi.org/10.1002/per.546

20. Spitzer RL, Kroenke K, Williams JW et al (2006) A brief measure for assessing generalized anxiety disorder: the gad-7. Arch Intern Med 166(10):1092-1097. https://doi.org/10.1001/archi nte.166.10.1092

21. Kuehnel V, Kollmeier B, Wagener K (1999) Entwicklung und Evaluation eines Satztests für die deutsche Sprache I. Design des Oldenburger Satztests 38:4-15

22. Brüggemann P, Szczepek AJ, Rose M et al (2016) Impact of multiple factors on the degree of tinnitus distress. Front Hum Neurosci 10:341. https://doi.org/10.3389/fnhum.2016.00341

23. Sanhueza I, Manrique-Huarte R, Calavia D et al (2019) Hearing impairment and quality of life in adults with asymmetric hearing loss: benefits of bimodal stimulation. J Int Adv Otol 15(1):62-69

24. Moteki H, Kitoh R, Tsukada K et al (2015) The advantages of sound localization and speech perception of bilateral electric acoustic stimulation. Acta Otolaryngol 135(2):147-153. https:// doi.org/10.3109/00016489.2014.951453

25. Kral A (2007) Unimodal and cross-modal plasticity in the 'deaf' auditory cortex. Int J Audiol 46(9):479-493. https://doi. org/10.1080/14992020701383027
26. Won JH, Schimmel SM, Drennan WR et al (2008) Improving performance in noise for hearing aids and cochlear implants using coherent modulation filteringa. Hear Res 239(1-2):1-11. https:// doi.org/10.1016/j.heares.2008.01.009

27. Olze H, Szczepek AJ, Haupt H et al (2011) Cochlear implantation has a positive influence on quality of life, tinnitus, and psychological comorbidity. Laryngoscope 121(10):2220-2227. https://doi. org/10.1002/lary.22145

28. Damen GWJA, Beynon AJ, Krabbe PFM et al (2007) Cochlear implantation and quality of life in postlingually deaf adults: longterm follow-up. Otolaryngol Head Neck Surg 136(4):597-604. https://doi.org/10.1016/j.otohns.2006.11.044

29. Knopke S, Szczepek AJ, Haussler SM et al (2017) Cochlear implantation of bilaterally deafened patients with tinnitus induces sustained decrease of tinnitus-related distress. Front Neurol 8:158. https://doi.org/10.3389/fneur.2017.00158

30. Smulders YE, Hendriks T, Eikelboom RH et al (2017) Predicting sequential cochlear implantation performance: a systematic review. Audiol Neurootol 22(6):356-363. https://doi. org/10.1159/000488386

Publisher's Note Springer Nature remains neutral with regard to jurisdictional claims in published maps and institutional affiliations. 